\title{
MEASURING CHANGE IN CHILDREN'S LEVELS OF PHYSICAL ACTIVITY OVER TIME
} USING A SELF-REPORT ASSESSMENT OF PHYSICAL ACTIVITY

\author{
N.K. Mullan ${ }^{1}$, J. Williams ${ }^{2}$, C. Glazebrook ${ }^{1}$ \\ ${ }^{1}$ Division of Psychiatry - Behavioural Sciences, ${ }^{2}$ Medical School, University of Nottingham, Nottingham, UK
}

Background and aims: Assessing the effectiveness of interventions to increase activity in children is challenging. Self report questionnaires of physical activity have advantages in terms of cost but may be limited by poor recall or other biases. This research aims to investigate the effectiveness of a self-report Physical Activity Questionnaire (PAQ) in monitoring change in levels of physical activity in children.

Methods: Participants were 55 children (27 boys, 28 girls) aged 9-11. Each child wore a NL-1000 accelerometer (New Lifestyles, USA) for three consecutive days. After each 24 hour period, total steps, distance travelled and activity minutes were recorded and the child completed the self report PAQ, which recalls activities completed in the previous 24 hour period; split into before, after and during school. Physical and sedentary activities were totalled.

Results: Of 155 invited to participate, $36.7 \%$ were recruited with $71 \%$ compliance. Mean PAQ physical activities scores decreased significantly between day 1 and day 2 and between day 2 and day 3. Similar pattern of change was observed in mean total activity minutes recorded on the accelerometer. There was a significant correlation between decline in activity minutes between day 1 and day 2 and decline over the same period in PAQ total activity scores $(\mathrm{r}=0.358)$. There was a statistical trend towards a similar relationship between change in activity minutes and change in PAQ scores between days 2 and $3(r=0.2)$.

Conclusion: The study provides some evidence that PAQ scores are sensitive to change in levels of physical activity in children. 